\title{
Reação de híbridos comerciais de milho às ferrugens polissora e tropical(1)
}

\author{
Renzo Garcia von Pinho(2), Magno Antônio Patto Ramalho(3), Ivan Carvalho Resende ${ }^{(4)}$,
} Herberte Pereira Silva ${ }^{(5)}$ e Gilberto Pozar ${ }^{(4)}$

\begin{abstract}
Resumo - Os objetivos deste trabalho foram avaliar o nível de resistência de oito híbridos comerciais de milho aos patógenos Puccinia polysora Underw e Physopella zeae (Mains) Cummins e Ramachar, e comparar a eficiência dos métodos da área abaixo da curva de progresso da doença (AACPD) e dos parâmetros de estabilidade fenotípica, na avaliação dessa resistência. Em quatro ambientes nas regiões Sudeste e Centro-Oeste do Brasil foram realizadas cinco avaliações da severidade das doenças, em intervalos de dez dias a partir dos 60 dias após a semeadura, utilizando uma escala diagramática com notas. Os parâmetros de estabilidade fenotípica estudados foram o coeficiente de regressão linear (b) entre a época de avaliação (x) e a severidade da doença (y) e o coeficiente de determinação $\left(\mathrm{R}^{2}\right)$. No caso de $P$. polysora, ambos os métodos utilizados mostraram-se eficientes na discriminação do nível de resistência dos híbridos, permitindo a classificação de modo semelhante. Quanto a P. zeae, não houve boa concordância entre os dois métodos, especialmente porque a discriminação do nível de resistência entre híbridos não foi expressiva. Os híbridos mais resistentes a P. polysora foram Z 8392, C 909 e C 333, e os mais suscetíveis, P 3069, AG 9012 e C 956. Os destaques, em termos de resistência a $P$. zeae, foram C 909 e C 333, e os híbridos mais suscetíveis, P 3069 e AG 9012
\end{abstract}

Termos para indexação: Puccinia polysora, Physopella zeae, ferrugem, resistência a doenças.

\section{Reaction of commercial corn cultivars to southern and tropical rusts}

\begin{abstract}
The goals of this work were to evaluate the resistance level of eight commercial corn hybrids to the pathogens Puccinia polysora Underw and Physopella zeae (Mains) Cummins \& Ramachar and to compare the efficiency of the methods of the area under the disease progress curve (AUDPC) and of the phenotypical stability parameters in the evaluation of this resistance. Experiments were conducted in four environments in the Southeast and Center-West regions of Brazil. Five evaluations for the severity of the diseases were performed at ten-day intervals from the 60th day after sowing, by employing a diagrammatic scale. The area under the disease progress curve (AUDPC) and also phenotypical stability parameters, i. e., the linear regression coefficient (b) between the evaluation time (x) and the disease severity (y) and the determination coefficient $\left(\mathrm{R}^{2}\right)$ were evaluated. It was found that in the case of $P$. polysora, both methods utilized proved to be effective to discriminate the resistance level of the hybrids, enabling ranking them in a similar way. To $P$. zeae, there was not good agreement between both methods, mainly because the discrimination of resistance level was not outstanding. The most resistant hybrids to P. polysora were Z 8392, C 909 and C 333, and the most susceptible were P 3069 , AG 9012 and C 956. The most outstanding ones in terms of resistance to $P$. zeae were $\mathrm{C} 909$ and $\mathrm{C} 333$ and the most susceptible were P 3069 and AG 9012
\end{abstract}

Index terms: Puccinia polysora, Physopella zeae, rusts, disease resistance.

(1) Aceito para publicação em 9 de junho de 2000

Extraído da tese de doutorado apresentada pelo primeiro autor à Universidade Federal de Lavras (UFLA), Lavras, MG. Financiado pela FAPEMIG

(2)UFLA, Dep. de Agricultura, Caixa Postal 37, CEP 37200-000 Lavras, MG. E-mail: renzo@ufla.br
(3)UFLA, Dep. de Biologia, Caixa Postal 37, CEP 37200-000 Lavras, MG. E-mail: magnoapr@ufla.br

(4)Sementes Monsanto Ltda, R. Eduardo Oliveira 940,

CEP 38400-174 Uberlândia, MG.

E-mail: ivan.c.resende@monsanto.com, gilberto.pozar@monsanto.com

(5)Zeneca Sementes Ltda, Caixa Postal 81, CEP 14140-000

Cravinhos, SP. E-mail: heberte.silva@brspz.zeneca.com 


\section{Introdução}

Nos últimos anos, a importância dos patógenos que infectam a cultura do milho tem aumentado, o que constitui um dos principais entraves para o contínuo aumento na produtividade da cultura, principalmente em semeaduras mais tardias. No Brasil, há pelo menos 20 patógenos que ocorrem na cultura e que podem causar prejuízos expressivos (Agroceres, 1996; Reis \& Casa, 1996). Entretanto, a ferrugempolissora, causada pelo fungo Puccinia polysora Underw, e a ferrugem-tropical, causada por Physopella zeae (Mains) Cummins \& Ramachar, são as que merecem maior destaque, especialmente nas regiões Centro-Oeste e Sudeste do País.

Embora as informações a respeito de cultivares com resistência a esses patógenos sejam ainda incipientes, sabe-se que o uso de cultivares resistentes constitui o método mais eficiente, racional e econômico para evitar ou ao menos diminuir os danos causados por esses patógenos. A disponibilidade de cultivares resistentes à ferrugem-polissora é maior do que para a ferrugem-tropical. É possível que isto se deva à ocorrência mais recente da ferrugem-tropical no Brasil. Como os programas de melhoramento de milho são muito dinâmicos, e produzem um grande número de novas cultivares anualmente, é necessário avaliar o comportamento desses materiais em relação a essas ferrugens, não só para direcionar futuros trabalhos de melhoramento visando à obtenção de cultivares resistentes, mas também para orientar na escolha e recomendação de cultivares para as diferentes regiões do País.

O uso da resistência horizontal tem sido mais eficiente no controle das ferrugens-do-milho (Robinson, 1987; Smith \& White, 1988; Parlevliet, 1993 ) porque esses patógenos são parasitas obrigatórios de grande variabilidade, o que dificulta a eficácia da resistência vertical. De $P$. polysora, são conhecidas 13 raças, e de $P$. zeae já foram identificadas duas raças (Robert, 1962; Yeh, 1986).

A quantificação da resistência horizontal pode ser realizada por meio da área abaixo da curva de progresso da doença (Leonard \& Fry, 1986; Bailey et al., 1987; Campbell \& Madden, 1990) ou pelo emprego de equações de regressão linear que permitem obter informações sobre a taxa de progresso da doença durante o desenvolvimento da cultura. Neste caso, o uso de métodos relativos à quantificação da estabilidade fenotípica, comumente empregado para a avaliação de outras características agronômicas, pode ser bem-sucedido (Pereira et al., 1996).

Os objetivos deste trabalho foram avaliar a resistência de oito híbridos comerciais de milho às ferrugens polissora e tropical e comparar a eficiência dos métodos da área abaixo da curva de progresso da doença e da estabilidade fenotípica na avaliação dessa resistência.

\section{Material e Métodos}

Os experimentos foram conduzidos em quatro ambientes de três locais das regiões Sudeste e Centro-Oeste do Brasil. Dois deles foram instalados na estação experimental da Cargill Agrícola S.A., localizada no Município de Santa Helena de Goiás, GO, a $17^{\circ} 80^{\prime}$ de latitude Sul e a $562 \mathrm{~m}$ de altitude. A semeadura foi realizada em 5 de novembro e em 27 de dezembro de 1996, respectivamente. As colheitas realizaram-se em 15 de abril de 1997, no primeiro experimento, e em 24 de maio de 1997 no segundo. Em Capinópolis, $\mathrm{MG}$, localizado a $18^{\circ} 60^{\prime}$ de latitude Sul e a $564 \mathrm{~m}$ de altitude, foi conduzido um experimento em área da empresa Sementes Agroceres S.A., com a semeadura realizada em 4 de novembro de 1996, e a colheita, em 5 de abril de 1997. Finalmente, instalou-se um experimento em 17 de dezembro de 1996, na estação experimental da Zeneca Agrícola S.A., localizada no Município de Cravinhos, SP, a $21^{\circ} 60^{\prime}$ de latitude Sul e a $564 \mathrm{~m}$ de altitude, cuja colheita se deu em 4 de abril de 1997.

Foram utilizados oito híbridos comerciais pertencentes às empresas: Sementes Agroceres S.A. (AG 9012, AG 5011), Cargill Agrícola S.A. (C 909, C 956 e C 333), Zeneca Agrícola S.A. (Z 8392 e Z 8440), e Pioneer Sementes Ltda (P 3069)

Em todos os experimentos utilizou-se o delineamento de blocos casualizados, com três repetições. As parcelas eram constituídas de quatro fileiras de $5,0 \mathrm{~m}$ de comprimento, sendo as duas fileiras centrais consideradas como úteis. O espaçamento entre fileiras foi de $0,9 \mathrm{~m}$, e a densidade foi de cinco plantas por metro linear, após o desbaste. Em uma das extremidades de cada repetição, foi colocada uma bordadura, com fileiras de 1,0 m de comprimento, com um híbrido suscetível às duas ferrugens (P 3069) que foi semeado 20 dias antes da instalação dos experimentos. Na outra extremidade das parcelas foi instalada outra bordadura, nas mesmas dimensões da anterior, porém com um híbrido resistente (C 333). 
A adubação de semeadura foi de $400 \mathrm{~kg} / \mathrm{ha}$ da fórmula $4(\mathrm{~N}): 20\left(\mathrm{P}_{2} \mathrm{O}_{5}\right): 20\left(\mathrm{~K}_{2} \mathrm{O}\right)+0,5 \% \mathrm{Zn}$. Em cobertura, foram aplicados $100 \mathrm{~kg}$ de N/ha na forma de uréia, distribuídos igualmente, em duas épocas: a primeira, quando as plantas estavam no estádio de quatro a cinco folhas, e a segunda, no estádio de sete a oito folhas. Durante a condução dos experimentos, foram empregados todos os tratos culturais recomendados para a cultura. Aos 30 dias após a emergência, foi feita uma inoculação, nas plantas da bordadura, de uma suspensão de esporos $\left(4 \times 10^{4}\right.$ esporos por $\mathrm{mL}$ ) de uma mistura de $P$. polysora e $P$. zeae. $O$ inóculo foi obtido de plantas infectadas, coletadas no local de condução dos experimentos

A avaliação da severidade de cada doença foi realizada por meio de uma escala de notas: $1,2,3,4,5,6,7,8$ e 9 , correspondente a: $0,0,5,10,30,50,70,80,90$ e $100 \%$, respectivamente, da área foliar afetada.

Foram feitas cinco a seis avaliações das severidades das doenças, dependendo do local, em intervalos de 10 dias, a partir dos 60 dias após a semeadura. Esses dados foram utilizados na análise de estabilidade e para calcular a área abaixo da curva de progresso da doença, conforme Campbell \& Madden (1990). Em relação aos dados da área abaixo da curva de progresso da doença (AACPD), foram realizadas, inicialmente, análises de variância em cada experimento. Posteriormente, realizaram-se análise de variância conjunta envolvendo os quatro experimentos e análises de variância das diferentes épocas de avaliação em um mesmo experimento.

Para as estimativas dos parâmetros de estabilidade foi utilizado o método proposto por Eberhart \& Russell (1966). Para isto, as diferentes épocas de avaliação das doenças foram consideradas como ambientes diferentes, e as análises feitas separadamente com relação a cada experimento.

Foram obtidas, também, estimativas de correlação classificatória de Spearman (Steel \& Torrie, 1980), entre as estimativas da AACPD dos diferentes experimentos, considerando cada doença separadamente e também entre a AACPD e a severidade média de doença nas diferentes épocas de avaliação (notas), e os coeficientes de regressão linear $\left(b_{1}\right)$ obtidos na análise de estabilidade.

\section{Resultados e Discussão}

Pela análise de variância conjunta da AACPD, envolvendo os quatro experimentos, constataram-se diferenças significativas ( $\mathrm{P} \leq 0,01)$ entre os híbridos, experimentos e na interação híbridos $\mathrm{x}$ experimen- tos. Isto mostra que ocorreu variabilidade entre os oito híbridos na resposta à infecção das ferrugens.

Apesar da significância da interação híbridos $\mathrm{x}$ experimentos, a coincidência no comportamento das cultivares nos diferentes experimentos, avaliado pelo coeficiente de correlação de Spearman, foi alta e significativa, em todas as combinações possíveis entre os experimentos, especialmente em relação a P. zeae, (Tabela 1). No caso de P. polysora, os valores destas correlações foram menores que os obtidos em relação a $P$. zeae, e não-significativo, em relação à metade das combinações possíveis entre os experimentos. Isto pode ser explicado pela menor severidade de $P$. polysora em alguns experimentos, o que dificultou a discriminação dos híbridos, reduzindo as estimativas das correlações obtidas.

Menores estimativas da correlação classificatória de Spearman seriam esperadas se as raças dos patógenos variassem entres os locais. A ocorrência de variabilidade desses fungos já foi constatada em outros países (Storey \& Ryland, 1954; Robert, 1962; Ullstrup, 1965; Yeh, 1986). As estimativas da correlação de Spearman em relação a $P$. polysora, entre os experimentos de Cravinhos, e o segundo experimento realizado em Santa Helena (Santa Helena 2) e Santa Helena 1 e Capinópolis, não foram significativas. Pelo menos em princípio, esse resultado possibilita inferir sobre a possível ocorrência de diferentes raças desse fungo nesses locais.

A magnitude da severidade das duas doenças foi suficiente para discriminar os híbridos resistentes em

Tabela 1. Coeficiente de correlação de Spearman da área abaixo da curva de progresso de doença em relação às ferrugens tropical (Physopella zeae) e polissora (Puccinia polysora), em oito híbridos de milho avaliados em quatro experimentos.

\begin{tabular}{lcc}
\hline Local & $P$. zeae & $P$. polysora \\
\hline Cravinhos x Santa Helena 1 & $0,88^{* *}$ & $0,90^{* *}$ \\
Cravinhos x Santa Helena 2 & $0,95^{* *}$ & $0,51^{\mathrm{ns}}$ \\
Cravinhos x Capinópolis & $0,71^{*}$ & $0,83^{* *}$ \\
Santa Helena 1 x Santa Helena 1 & $0,81^{* *}$ & $0,69^{\mathrm{ns}}$ \\
Santa Helena 1 x Capinópolis & $0,81^{*}$ & $0,90^{* *}$ \\
Santa Helena 2 x Capinópolis & $0,69^{*}$ & $0,62^{\mathrm{ns}}$ \\
\hline
\end{tabular}

ns Não-significativo. ${ }^{*} \mathrm{e}^{* *}$ Significativo a $5 \%$ e $1 \%$ de probabilidade, respectivamente pelo teste $\mathrm{F}$. 
todos os experimentos (Tabela 2). A severidade da ferrugem-tropical foi maior em todos os experimentos. Em Santa Helena 2, por exemplo, ela foi mais do dobro da ferrugem-polissora. O experimento onde ocorreu a maior severidade da ferrugem-polissora foi o de Cravinhos, com valor da AACPD de 155,96, e o de menor severidade foi o de Capinópolis, com valor da AACPD de 91,25.

Apesar da maior severidade de ferrugem-tropical em todos os experimentos, os valores extremos da AACPD foram menores em relação a esse patógeno (Tabela 2). Isto mostra que, embora exista variabilidade da resistência, esta não é tão expressiva como a observada em relação a P. polysora. É possível que isto se deva ao fato de que a importância desta doença é muito recente, no Brasil, o que não permitiu ainda o desenvolvimento de cultivares com alto nível de resistência.
Com relação à $\mathrm{AACPD}$, os híbridos mais resistentes a P. polysora foram o Z 8392, C 909 e C 333, e os mais suscetíveis, os híbridos P 3069, AG 9012, C 956 (Tabela 2). O comportamento dos híbridos avaliados foi coincidente em todos os experimentos, o que pode ser confirmado pelos altos valores dos coeficientes de correlação de Spearman (Tabela 1). Esses resultados são coincidentes com informações disponíveis pelas empresas detentoras de algumas dessas cultivares (Agroceres, 1996; Cargill, 1996).

No caso de $P$. zeae, os híbridos mais resistentes foram também o C 909 e o C 333. Já o híbrido C 956, que para o outro patógeno foi um dos mais suscetíveis, a $P$. zeae, esteve entre os mais resistentes. O híbrido Z 8392, que foi o mais resistente a P. polysora, não apresentou o mesmo comportamento ante a ferrugem-tropical. Os híbridos P 3069 e AG 9012 estiveram novamente entre os materiais com

Tabela 2. Área abaixo da curva de progresso da doença (AACPD) obtida em relação à severidade da ferrugem-polissora (Puccinia polysora) e da ferrugem-tropical (Physopella zeae) nos quatro experimentos, em oito cultivares de milho ${ }^{(1)}$.

\begin{tabular}{lccccc}
\hline Cultivar & Cravinhos & Santa Helena 1 & Santa Helena 2 & Capinópolis & Média \\
\hline \multicolumn{5}{c}{ Puccinia polysora } \\
AG 9012 & $186,67 \mathrm{~b}$ & $180,00 \mathrm{~b}$ & $170,33 \mathrm{c}$ & $128,33 \mathrm{c}$ & $166,33 \mathrm{~d}$ \\
AG 5011 & $178,33 \mathrm{~b}$ & $170,00 \mathrm{~b}$ & $110,00 \mathrm{c}$ & $111,67 \mathrm{~b}$ & $142,50 \mathrm{~d}$ \\
C 909 & $81,00 \mathrm{a}$ & $91,67 \mathrm{a}$ & $70,00 \mathrm{~b}$ & $65,00 \mathrm{a}$ & $76,92 \mathrm{~b}$ \\
C 956 & $213,33 \mathrm{~b}$ & $177,67 \mathrm{~b}$ & $103,33 \mathrm{c}$ & $103,33 \mathrm{~b}$ & $149,42 \mathrm{~d}$ \\
Z 8392 & $51,67 \mathrm{a}$ & $80,00 \mathrm{a}$ & $41,67 \mathrm{a}$ & $60,00 \mathrm{a}$ & $58,33 \mathrm{a}$ \\
Z 8440 & $186,67 \mathrm{~b}$ & $141,67 \mathrm{~b}$ & $65,00 \mathrm{~b}$ & $71,67 \mathrm{a}$ & $116,25 \mathrm{c}$ \\
C 333 & $78,33 \mathrm{a}$ & $95,00 \mathrm{a}$ & $78,33 \mathrm{~b}$ & $58,33 \mathrm{a}$ & $77,50 \mathrm{~b}$ \\
P 3069 & $271,67 \mathrm{c}$ & $221,67 \mathrm{c}$ & $101,67 \mathrm{c}$ & $131,67 \mathrm{c}$ & $181,67 \mathrm{e}$ \\
\hline Média & 155,96 & 144,71 & 92,54 & 91,25 & 121,12 \\
\hline C.V. $(\%)$ & 14,15 & 12,59 & 13,02 & 13,21 & 19,06 \\
\hline & & & & $185,88 \mathrm{~d}$ \\
AG 9012 & $210,00 \mathrm{c}$ & $192,50 \mathrm{c}$ & $223,33 \mathrm{c}$ & $117,67 \mathrm{~b}$ & $166,25 \mathrm{c}$ \\
AG 5011 & $168,33 \mathrm{~b}$ & $180,00 \mathrm{c}$ & $189,33 \mathrm{~b}$ & $118,33 \mathrm{~b}$ & $132,71 \mathrm{a}$ \\
C 909 & $123,33 \mathrm{a}$ & $137,50 \mathrm{a}$ & $178,33 \mathrm{a}$ & $91,67 \mathrm{a}$ & $154,00 \mathrm{~b}$ \\
C 956 & $171,67 \mathrm{~b}$ & $145,00 \mathrm{a}$ & $206,67 \mathrm{~b}$ & $92,67 \mathrm{a}$ & $172,75 \mathrm{c}$ \\
Z 8392 & $203,33 \mathrm{c}$ & $160,00 \mathrm{~b}$ & $219,33 \mathrm{~b}$ & $108,33 \mathrm{a}$ & $184,21 \mathrm{~d}$ \\
Z 8440 & $208,33 \mathrm{c}$ & $187,50 \mathrm{c}$ & $241,67 \mathrm{c}$ & $99,33 \mathrm{a}$ & $132,92 \mathrm{a}$ \\
C 333 & $145,00 \mathrm{a}$ & $150,00 \mathrm{a}$ & $146,67 \mathrm{a}$ & $90,00 \mathrm{a}$ & $210,88 \mathrm{e}$ \\
P 3069 & $257,67 \mathrm{c}$ & $212,50 \mathrm{~d}$ & $246,67 \mathrm{c}$ & $126,67 \mathrm{~b}$ & 167,17 \\
\hline Média & 185,96 & 170,63 & 206,50 & 105,58 & 10,70 \\
\hline C.V. $(\%)$ & 9,20 & 7,64 & 7,65 & 8,53 & \\
\hline
\end{tabular}

${ }^{(1)}$ Médias seguidas pelas mesmas letras, na coluna, não diferem entre si pelo teste de Scott Knott a 5\% de probabilidade. 
maior suscetibilidade (Tabela 2). Tais resultados sugerem a existência de controles genéticos parcialmente distintos em relação a cada patógeno.

Outra alternativa para se avaliar o nível de resistência de cultivares são os parâmetros de estabilidade fenotípica, já empregados com relação à soja (Pereira et al., 1996). Para isso, é necessário proceder às análises de variância envolvendo as diferentes épocas de avaliação da severidade (notas) de ocorrência dos patógenos. Constataram-se, de modo geral, diferenças significativas $(\mathrm{P} \leq 0,01)$ quanto às fontes de variação híbridos, às épocas de avaliação e à interação híbrido $\mathrm{x}$ épocas em todos os experimentos e em ambos os patógenos. As estimativas dos parâmetros de estabilidade pelo método de Eberhart \& Russell (1966) são apresentadas na Tabela 3 .

Quanto a $P$. polysora, o híbrido Z 8392 pode ser considerado o mais resistente, tendo em vista as estimativas de $b_{0}$ e $b_{1}$. A pequena estimativa de $b_{0}$ obtida, em relação a esse híbrido, em todos os experi- mentos, indica a menor severidade média desse patógeno. Já a pequena estimativa do coeficiente de regressão linear $\left(b_{1}\right)$, mostra o menor progresso da doença com o decorrer das épocas (Tabela 3).

No outro extremo, como material mais suscetível, está o híbrido P 3069, que apresentou estimativas elevadas, em todos os experimentos, tanto de $b_{0}$ como de $b_{1}$. Isto é, esse híbrido apresentou a maior severidade média da doença e com progresso da doença acima da média obtida em cada época de avaliação.

Como era esperado, ocorreu associação positiva e alta entre as estimativas de $b_{0}$ e $b_{1}$, exceto em Santa Helena 2, onde, como já mencionado, a severidade da ferrugem-polissora foi baixa (Tabela 3). Essa é uma situação favorável, pois pode-se utilizar tanto as estimativas de $b_{0}$ como de $b_{1}$, na discriminação das cultivares. Em princípio, o $b_{1}$ é mais informativo, pois possibilita inferir sobre a taxa de progresso da doença. Assim, $b_{1}$ superior a 1,0 indica que, para aquele híbrido, o progresso da doença foi acima da

Tabela 3. Médias da severidade da ferrugem-polissora (Puccinia polysora) e da ferrugem-tropical (Physopella zeae) $\left(b_{0}\right)$, coeficientes de regressão $\left(b_{1}\right)$ e coeficientes de determinação $\left(\mathrm{R}^{2}\right)$ dos híbridos de milho avaliados em cinco ou seis épocas nos quatro experimentos.

\begin{tabular}{|c|c|c|c|c|c|c|c|c|c|c|c|c|}
\hline \multirow[t]{2}{*}{ Cultivar } & \multicolumn{3}{|c|}{ Cravinhos } & \multicolumn{3}{|c|}{ Santa Helena 1} & \multicolumn{3}{|c|}{ Santa Helena 2} & \multicolumn{3}{|c|}{ Capinópolis } \\
\hline & $\mathrm{b}_{0}$ & $b_{1}$ & $\mathrm{R}^{2}$ & $\mathrm{~b}_{0}$ & $b_{1}$ & $\mathrm{R}^{2}$ & $\mathrm{~b}_{0}$ & $b_{1}$ & $\mathrm{R}^{2}$ & $\mathrm{~b}_{0}$ & $b_{1}$ & $\mathrm{R}^{2}$ \\
\hline & \multicolumn{12}{|c|}{ Puccinia polysora } \\
\hline AG 9012 & 4,08 & $1,38^{* *}$ & 99,04 & 4,27 & $1,28^{*}$ & 96,31 & 2,60 & 1,28 & 97,77 & 3,27 & $1,47^{* *}$ & 99,08 \\
\hline AG 5011 & 3,61 & $1,37^{* *}$ & 94,14 & 4,13 & 1,08 & 97,05 & 2,73 & 0,83 & 87,82 & 2,93 & $1,50^{* *}$ & 99,29 \\
\hline C 909 & 1,64 & $0,45^{\text {** }}$ & 96,44 & 2,33 & $0,70^{* *}$ & 95,50 & 1,80 & 1,24 & 98,63 & 1,73 & $0,71^{*}$ & 94,43 \\
\hline C 956 & 4,31 & $1,48^{* * *}$ & 98,96 & 4,30 & 1,06 & 95,62 & 2,53 & 0,87 & 68,58 & 2,60 & 0,94 & 97,41 \\
\hline Z 8392 & 1,06 & $0,06^{* * *}$ & 43,28 & 2,07 & $0,61^{* *}$ & 87,45 & 1,07 & $0,20^{*}$ & 70,26 & 1,53 & $0,48^{* *}$ & 90,59 \\
\hline Z 8440 & 3,69 & 1,03 & 97,08 & 3,40 & 0,88 & 92,83 & 1,73 & 1,05 & 80,52 & 1,87 & $0,73^{\text {*** }}$ & 90,12 \\
\hline C 333 & 1,61 & $0,40^{* * *}$ & 78,48 & 2,67 & 1,12 & 68,40 & 2,00 & 0,99 & 96,78 & 1,53 & $0,51^{* *}$ & 94,91 \\
\hline P 3069 & 5,41 & $1,93^{\text {** }}$ & 99,29 & 5,40 & $1,27^{*}$ & 96,79 & 2,46 & $1,55^{* *}$ & 97,42 & 3,40 & $1,65^{\text {** }}$ & 98,75 \\
\hline \multirow[t]{2}{*}{$\mathrm{r}\left(\mathrm{b}_{0} \times \mathrm{b}_{1}\right)^{(1)}$} & & 0,98 & & & 0,83 & & & 0,57 & & & 0,97 & \\
\hline & \multicolumn{12}{|c|}{ Physopella zeae } \\
\hline AG 9012 & 4,22 & 1,13 & 96,58 & 4,80 & 1,20 & 96,95 & 5,53 & 1,02 & 91,63 & 2,97 & 1,06 & 93,47 \\
\hline AG 5011 & 3,36 & $0,38^{* *}$ & 91,20 & 4,50 & $0,45^{*}$ & 90,27 & 5,00 & 0,94 & 91,66 & 2,93 & $1,53^{\text {*** }}$ & 97,94 \\
\hline С 909 & 2,56 & 0,93 & 91,85 & 3,40 & $1,28^{*}$ & 97,78 & 4,47 & $1,43^{*}$ & 93,11 & 2,27 & 0,97 & 95,02 \\
\hline C 956 & 3,44 & 1,27 & 99,31 & 3,60 & 1,05 & 90,36 & 5,27 & $1,43^{*}$ & 89,63 & 2,30 & 0,91 & 95,83 \\
\hline Z 8392 & 4,06 & 1,12 & 96,54 & 3,90 & 1,05 & 90,85 & 5,50 & 0,75 & 96,34 & 2,73 & $0,75^{*}$ & 93,44 \\
\hline Z 8440 & 4,17 & 0,91 & 98,08 & 4,70 & 1,17 & 91,57 & 6,00 & $0,51^{* *}$ & 81,99 & 2,50 & $0,77^{*}$ & 89,83 \\
\hline C 333 & 2,97 & 0,63 & 89,24 & 3,70 & $0,56^{*}$ & 87,27 & 3,67 & 0,72 & 98,59 & 2,27 & 1,12 & 97,14 \\
\hline P 3069 & 5,19 & $1,62^{* * *}$ & 97,85 & 5,20 & 1,24 & 89,41 & 6,07 & 1,20 & 89,25 & 3,17 & 0,88 & 96,97 \\
\hline $\mathrm{r}\left(\mathrm{b}_{0} \times \mathrm{b}_{1}\right)^{(1)}$ & & 0,67 & & & 0,16 & & & $-0,06$ & & & 0,20 & \\
\hline
\end{tabular}

${ }^{(1)}$ Coeficiente de correlação. ${ }^{*}$ e ${ }^{* *}$ Significativo a $5 \%$ e $1 \%$ de probabilidade, respectivamente 
média e portanto o material é mais suscetível, como já salientado.

Nos casos dos híbridos mais resistentes, os valores de $\mathrm{R}^{2}$ foram os mais baixos (Tabela 3 ). Em princípio, isso indica um pior ajuste dos dados à equação de regressão proposta. Contudo, deve ser enfatizado que nesses casos, como as notas foram sempre baixas, pequenas flutuações acarretam proporcionalmente maiores desvios no modelo, e em consequiência, menores valores de $\mathrm{R}^{2}$.

As estimativas dos parâmetros de estabilidade de P. zeae também são apresentadas na Tabela 3 . A discriminação dos híbridos, nesse caso, não foi tão evidente como no caso de P. polysora. Isso ocorreu porque os níveis de resistência dos híbridos avaliados não foram tão distintos. Porém, mesmo assim, o híbrido C 333 mostrou ser um dos mais resistentes, como já ocorrera com o uso da AACPD. No caso do híbrido C 909, as estimativas de $\mathrm{b}_{1}$, em pelo menos dois locais, foram acima da média e não permitem inferir que ele seja resistente ao patógeno em todos os ambientes. Os híbridos P 3069 e AG 9012 mostraram maiores estimativas de $\mathrm{b}_{0} \mathrm{em}$ todos os locais, e $b_{1}$, acima de 1,0 na maioria dos locais, e portanto podem ser considerados os mais suscetíveis, como já ocorrera com as estimativas da AACPD.

A existência da interação híbridos $\mathrm{x}$ épocas de avaliação mostrou que o comportamento dessas cultivares não foi coincidente nas diferentes épocas de avaliação, o que, de certa forma, complica o trabalho do melhorista. Isso implica a necessidade de efetuar mais de uma avaliação dessas doenças, aumentando, conseqüentemente, o trabalho de avaliação. Utilizando esses dados para a discriminação da resistência, as opções são os métodos da AACPD ou as estimativas dos parâmetros de estabilidade. No presente trabalho, as estimativas da correlação classificatória quanto à resistência dos híbridos obtida pelos dois métodos foi positiva e alta, em todos os locais, no caso de $P$. polysora, indicando, assim, que ambas podem ser utilizadas (Tabela 4). No caso de $P$. zeae, a correlação foi praticamente 1,0 entre AACPD e $b_{0}$, porém praticamente zero no caso da AACPD e $b_{1}$, o que indica que tratando-se desse patógeno, não houve coincidência na avaliação do progresso da doença pelos dois métodos. Novamente, merece ser destacado que, no caso desse patógeno,
Tabela 4. Coeficiente de correlação de Spearman entre a área abaixo da curva de progresso da doença (AACPD) e a severidade média de doença $\left(b_{0}\right)$ e os coeficientes de regressão $\left(b_{1}\right)$ obtidos na análise de estabilidade considerando os oito híbridos de milho avaliados nos quatro experimentos.

\begin{tabular}{lcccc}
\hline Correlação & Cravinhos & $\begin{array}{c}\text { Santa } \\
\text { Helena 1 }\end{array}$ & $\begin{array}{c}\text { Santa } \\
\text { Helena 2 }\end{array}$ & Capinópolis \\
\hline \multicolumn{4}{c}{ Puccinia polysora } \\
AACPD x b 0 & $0,99^{* *}$ & $0,98^{* *}$ & $0,98^{* *}$ & $0,99^{* *}$ \\
AACPD x b 1 & $0,96^{* *}$ & $0,83^{* *}$ & $0,26^{\mathrm{ns}}$ & $0,95^{* *}$ \\
\hline \multicolumn{4}{c}{ Physopella zeae } \\
AACPD x b b & $1,00^{* *}$ & $1,00^{* *}$ & $1,00^{* *}$ & $0,97^{* *}$ \\
AACPD x b & $0,62^{\mathrm{ns}}$ & $0,15^{\mathrm{ns}}$ & $-0,07^{\mathrm{ns}}$ & $-0,10^{\mathrm{ns}}$ \\
\hline
\end{tabular}

ns Não-significativo ${ }^{* *}$ Significativo a $1 \%$ de probabilidade

a discriminação dos híbridos utilizados não foi tão evidente, o que deve ter contribuído para a não-concordância entre os procedimentos.

\section{Conclusões}

1. No caso de P. polysora, tanto a área abaixo da curva de progresso da doença quanto os parâmetros da estabilidade fenotípica são eficientes para a discriminação do nível de resistência dos híbridos, e permitem a classificação de modo semelhante.

2. Quanto a $P$. zeae, não há boa concordância entre os dois métodos empregados para a avaliação da resistência, especialmente porque a discriminação no nível de resistência dos híbridos não é expressiva.

3. O nível de resistência entre as cultivares avaliadas é variável e independente das duas doenças consideradas.

\section{Referências}

AGROCERES (São Paulo, SP). Guia Agroceres de sanidade. São Paulo : Sementes Agroceres, 1996. 72 p.

BAILEY, B. A.; SCHUH, W.; FREDERIKSEN, R. A.; BOCKHOLT, A. J.; SMITH, J. D. Identification of "slow rusting" resistance to Puccinia polysora in maize inbreds and single crosses. Plant Disease, St. Paul, v. 71, n. 6, p. 518-521, June 1987.

CAMPBELL, C. L.; MADDEN, L. V. Monitoring epidemics: diseases. In Introduction to plant 
disease epidemiology. New York : J. Wiley, 1990. p. 107 128.

CARGILL (São Paulo, SP). Guia técnico Cargill. São Paulo : Cargill Agrícola, 1996. 103 p.

EBERHART, S. A.; RUSSELL, W. A. Stability parameters for comparing varieties. Crop Science, Madison, v. 6, p. 36-40, 1966.

LEONARD, K. J.; FRY, W. E. (Ed.). Plant disease epidemiology. New York : Macmillan, 1986. 372 p

PARLEVLIET, J. E. What is durable resistance: a general outline. In: JACOBS, T. H.; PARLEVLIET, J. E. (Ed.). Durability of disease resistance. Dordrecht Kluwer Academic, 1993. p. 23-39.

PEREIRA, D. G.; SEDIYAMA, T.; CRUZ, C. D.; GOMES, J. L.; TEIXEIRA, R. C. Análise da estabilidade fenotípica de cultivares de soja (Glycine $\max (\mathrm{L}$.) Merril) em relação a diferentes isolados de Diaphorte phaseolorum f. $\mathrm{sp}$ meridionalis. Revista Ceres, Viçosa, v. 43, n. 250 p. $743-754,1996$.

REIS, E. M.; CASA, R. T. Manual de identificação e controle de doenças de milho. Passo Fundo : Aldeia Norte, 1996. 80 p.
ROBERT, A. L. Host ranges and races of the corn rusts. Phytopathology, St. Paul, v. 52, p. 1010-1017, Oct. 1962.

ROBINSON, R. A. Host management in crop pathosystems. New York : Macmillan, 1987. 263 p.

SMITH, D. R.; WHITE, D. G. Diseases of corn. In: SPRAGUE, G. F.; DUDLEY, J. W. (Ed.). Corn and corn improvement. 3. ed. Madison : American Society of Agronomy Press, 1988. p. 687-766.

STEEL, R. G.; TORRIE, J. K. Principles and procedures of statistics: a biometrical approach. 2. ed. Tokyo : McGraw-Hill, 1980. 633 p.

STOREY, H. J.; RYLAND, A. K. Resistance to the maize rust Puccinia polysora. Nature, London, v. 173, p. 778-779, Apr. 1954.

ULLSTRUP, A. J. Inheritance and linkage of a gene determining resistance in maize to an American race to Puccinia polysora. Phytopathology, St. Paul, v. 55, p. 425-428, Apr. 1965.

YEH, C. C. Studies on rusts of maize. Journal of Agricultural Research of China, Wufeng, v. 35, n. 1, p. 81-93, 1986. 